\title{
Charge stripes and spin correlations in copper-oxide superconductors
}

\author{
J. M. Tranquada ${ }^{\mathrm{a} *}$

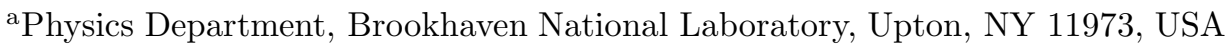

Recent neutron diffraction studies have yielded evidence that, in a particular cuprate family, holes doped into the $\mathrm{CuO}_{2}$ planes segregate into stripes that separate antiferromagnetic domains. Here it is shown that such a picture provides a quantitatively consistent interpretation of the spin fluctuations measured by neutron diffraction in $\mathrm{La}_{1.85} \mathrm{Sr}_{0.15} \mathrm{CuO}_{4}$ and $\mathrm{YBa}_{2} \mathrm{Cu}_{3} \mathrm{O}_{6+x}$.

\section{INTRODUCTION}

To obtain superconductivity in a layered copper-oxide compound, it is necessary to introduce charge carriers into the antiferromagnetic $\mathrm{CuO}_{2}$ planes. Recent neutron diffraction studies of the system $\mathrm{La}_{1.6-x} \mathrm{Nd}_{0.4} \mathrm{Sr}_{x} \mathrm{CuO}_{4}$ [1].2 provide evidence that the dopant-induced holes choose to segregate into periodically-spaced stripes which separate antiferromagnetic domains, in a manner similar to that found in hole-doped $\mathrm{La}_{2} \mathrm{NiO}_{4}$ [3]. The charge and spin stripe modulations are identified by the appearance of scattering at incommensurate positions. In the Nd-doped system, elastic scattering is observed, corresponding to static stripes. In pure $\mathrm{La}_{2-x} \mathrm{Sr}_{x} \mathrm{CuO}_{4}$, the magnetic scattering that is observed is purely inelastic 泊. Where samples with and without Nd, but with the same $\mathrm{Sr}$ concentration, have been measured, the incommensurate (IC) splittings of the magnetic signal are found to be essentially identical [2, [4. 5]. It has been proposed that the spin correlations in the two systems are fundamentally the same, thus implying similar charge correlations. The static nature of the stripes in the Nd-doped system is attributed [1] to pinning of the otherwise dynamic correlations by a special distortion of the lattice [6]. That distortion is driven by purely ionic interactions and is stabilized by the smaller ionic radius of the substituted Nd.

To strengthen the case for the charge-stripe in-

\footnotetext{
*Work at Brookhaven is supported by Contract No. DEAC02-76CH00016, Division of Materials Sciences, U.S. Department of Energy.
}

terpretation, it is necessary go beyond a simple comparison of incommensurabilities. In this paper I will show that the neutron scattering measurements of spin fluctuations in superconducting $\mathrm{La}_{1.85} \mathrm{Sr}_{0.15} \mathrm{CuO}_{4}$ and $\mathrm{YBa}_{2} \mathrm{Cu}_{3} \mathrm{O}_{6.6}$ are quantitatively consistent with what one should expect based on the stripe picture. The general expectations are described in section 2. Specific analyses for the two different superconductors are given in sections 3 and 4 , respectively. Section 5 contains some further discussion.

\section{MODEL}

First recall the situation for the 2D Heisenberg model [7]. At $T=0$, where long-range order exists, the spin-wave dispersion is linear at small $\mathbf{q}$ $\left(=\mathbf{Q}-\mathbf{Q}_{\mathrm{AF}}\right)$, and can be expressed as $\omega=c_{0} q$. At finite temperatures, thermal fluctuations result in a finite spin-spin correlation length $\xi$. Spin waves are overdamped for $q<1 / \xi$, but spin-wave dispersion should still be detectable for $q>1 / \xi$ (as long as $k T$ is less than the superexchange energy $J$ ). The characteristic energy $\Gamma_{0}$ separating the over- and underdamped regimes is then given by

$\Gamma_{0} \approx \hbar c_{0} / \xi$.

As for the dynamical susceptibility, $\chi^{\prime \prime}(\mathbf{Q}, \omega), \Gamma_{0}$ acts somewhat like a gap energy [7]. If $\chi^{\prime \prime}$ is integrated over $\mathbf{Q}$ to give $\tilde{\chi}^{\prime \prime}(\omega)$, then there is a peak at $\hbar \omega \approx \Gamma_{0}$, with $\tilde{\chi}^{\prime \prime}(\omega)$ going to zero as $\omega$ goes to zero.

Next consider an ordered stripe phase. The charge stripes need not affect the superexchange 
energy within the antiferromagnetic domains, but they certainly will weaken the magnetic coupling between domains. Castro Neto and Hone [8] have considered the effect of the anisotropic coupling on the spin wave velocity. For a large anisotropy, the velocity $c$ for propagation parallel to the stripes is equal to $c_{0} / \sqrt{2}$; it is more difficult to estimate an effective velocity perpendicular to the stripes because the stripe modulation should result in several optical branches as well as the acoustic mode. An experimental test of this situation is given by recent measurements of spin waves in stripe-ordered $\mathrm{La}_{2} \mathrm{NiO}_{4.133}$ [9]. That study found $c=0.6 c_{0}$ for propagation parallel to the stripes. Dispersion was less well defined but comparable in the perpendicular direction. (Note that the spin waves disperse out of the IC points in reciprocal space that characterize the magnetic order.) Thus, it seems reasonable to take $c=c_{0} / \sqrt{2}$ as an estimate of the effective spin-wave velocity in an ordered stripe phase.

Finally, consider a stripe phase with a finite spin-spin correlation length induced by fluctuations of the charge stripes. The characteristic energy separating over- and underdamped modes should now be

$\Gamma \approx \hbar c_{0} / \sqrt{2} \xi$.

Excitations at $\hbar \omega>\Gamma$ should look spin-wave-like. On the other hand, a scan at a fixed excitation energy smaller than $\Gamma$ through the IC points characterizing the stripe correlations should show a broad peak centered at each IC point, with the width varying inversely as $\xi$. To obtain an analytic formula describing the $q$-dependence of the scattering, consider a model in which the spins have a uniform correlation within each domain, but where the correlations between domains fall off exponentially with distance. If the charge stripes are separated by $n$ lattice spacings, then the intensity along $\mathbf{Q}=\left(h, \frac{1}{2}, 0\right)$ should be proportional to

$I(h) \sim|F|^{2} \frac{1-p^{2}}{1+p^{2}-2 p \cos (2 \pi n h)}$,

where $p=(-1)^{n+1} \exp (-n a / \xi)$, and $F$ is the structure factor for a single domain. (The factor of -1 included in $p$ takes care of the antiphase relationship between neighboring domains.) For the calculations below, it will be assumed that the spin correlations also fall off exponentially parallel to the stripes, with the same correlation length $\xi$.

\section{3. $\mathrm{La}_{1.85} \mathrm{Sr}_{0.15} \mathrm{CuO}_{4}$}

Recently, Hayden et al. 10] reported inelastic neutron scattering measurements of $\chi^{\prime \prime}(\mathbf{Q}, \omega)$ in $\mathrm{La}_{1.86} \mathrm{Sr}_{0.14} \mathrm{CuO}_{4}$, covering a very large energy range. They found that $\chi^{\prime \prime}$ at $\hbar \omega>100 \mathrm{meV}$ could be modelled reasonably well using spin wave formulas, with only a slight reduction in $J$. At lower energies, they showed that $\tilde{\chi}^{\prime \prime}(\omega)$ has a peak at $\Gamma=(22 \pm 5) \mathrm{meV}$. If this latter result is combined with the value $\hbar c_{0}=(850 \pm 30) \mathrm{meV}$ $\AA$ [11], then by inverting Eq. (2) one obtains the estimate $\xi=(27 \pm 6) \AA=(7.0 \pm 1.5) a$.

Figure 1 shows an inelastic scan, at a fixed energy transfer of $3 \mathrm{meV}$, through the IC peaks found in a single crystal of $\mathrm{La}_{1.85} \mathrm{Sr}_{0.15} \mathrm{CuO}_{4}$ [12]. The peak positions are consistent with a charge stripe period of $n=4$. The solid line through the data points is a calculation using Eq. (3) and the estimate $\xi=7 a$ - only the amplitude and the linear background have been adjusted to fit the data. (The calculated intensity, taking into account signal from all 4 IC peaks, was also convolved with the experimental resolution function.) The calculation clearly gives an excellent description of the data.

\section{4. $\mathrm{YBa}_{2} \mathrm{Cu}_{3} \mathrm{O}_{6.6}$}

In contrast to the 214 system, measurements of $\chi^{\prime \prime}$ in $\mathrm{YBa}_{2} \mathrm{Cu}_{3} \mathrm{O}_{6.6}$ do not show well resolved IC peaks [13]. As shown in Fig. 2, the scattered intensity is peaked at $\mathbf{Q}_{\mathrm{AF}}$, but with a peak shape that has a rather flat top and steep sides. Despite the lack of clear evidence for incommensurability, it is possible nevertheless to describe the data with the same stripe model discussed above. To give the stripe picture an honest test, $\xi$ will again be estimated using Eq. (2). The value of $\Gamma$ is approximately $(30 \pm 5) \mathrm{meV}$ [13], and $\hbar c_{0}=(670 \pm 30) \mathrm{meV}-\AA$ [14,15], which together yield $\xi=(16 \pm 3) \AA=(4.1 \pm 0.8) a$. To estimate 


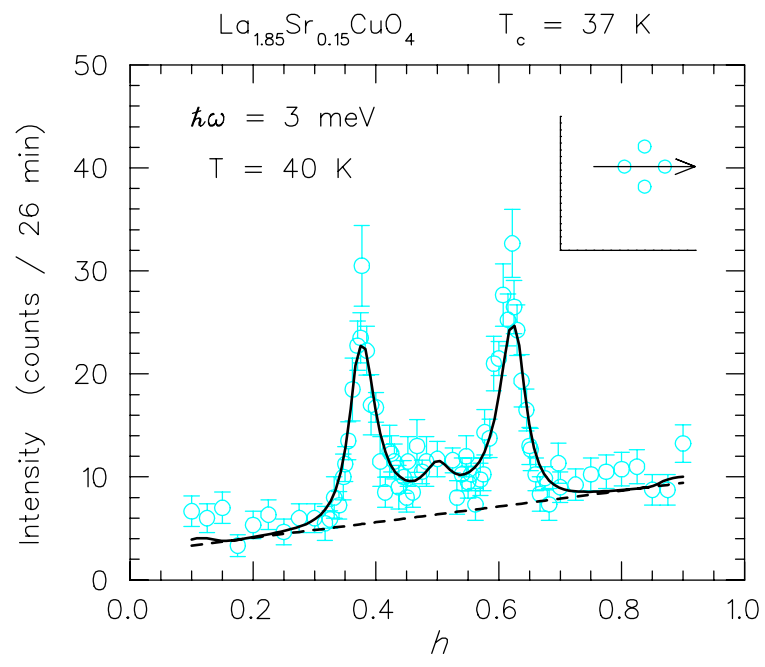

Figure 1. Inelastic scan at $\hbar \omega=3 \mathrm{meV}$ through the incommensurate points (see inset) measured at $T=40 \mathrm{~K}$ on a crystal of $\mathrm{La}_{1.85} \mathrm{Sr}_{0.15} \mathrm{CuO}_{4}$ with $T_{c}=37 \mathrm{~K}$. Solid line is the calculation described in the text; dashed line is the fitted background. Scan is along $\mathbf{Q}=\left(h, \frac{1}{2}, 0\right)$, within the $(h k 0)$ zone, as indicated schematically in the inset.

the stripe spacing, assuming that the hole density within the charge stripes is the same as in the 214 system [1], it is first necessary evaluate the hole concentration within a plane. Inverting the formula 16.

$T_{c} / T_{c, \max }=1-82.6(p-0.16)^{2}$,

yields $p \approx 0.088$ for the $T_{c}=53-\mathrm{K}$ sample, which corresponds closely to $n=6$. The solid line through the data in Fig. 2 represents a calculation using the same stripe model as above, with the parameter values $n=6$ and $\xi=4.1 a$. The calculated function was convolved with the spectrometer resolution function, and only the amplitude and background were adjusted to fit the data.

For the calculated curve, the filling in of weight between the IC peaks occurs because $\xi$ is less than the stripe spacing na. In this case the weak correlation between neighboring antiphase domains

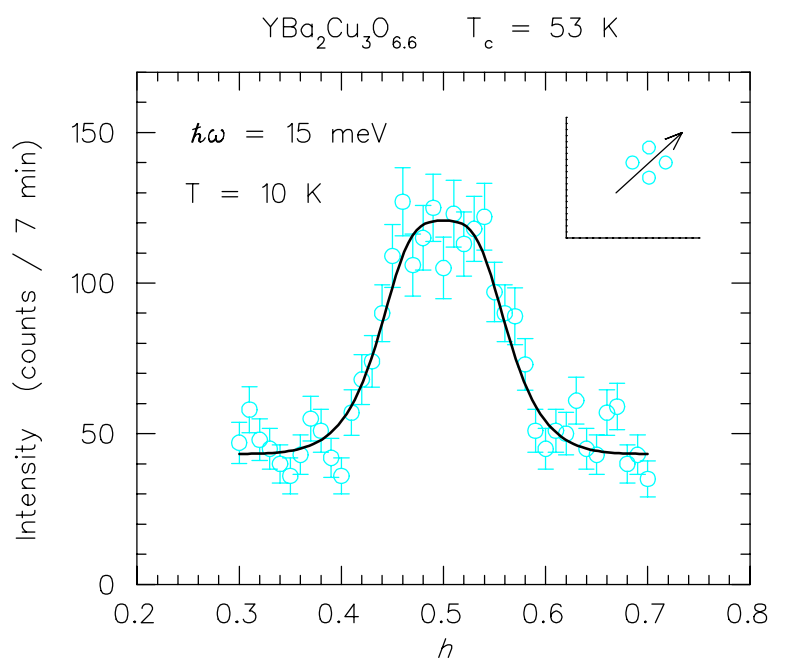

Figure 2. Inelastic scan at $\hbar \omega=15 \mathrm{meV}$ along the zone diagonal (see inset) measured at $T=10 \mathrm{~K}$ on a crystal of $\mathrm{YBa}_{2} \mathrm{Cu}_{3} \mathrm{O}_{6.6}$ with $T_{c}=53 \mathrm{~K}$. The solid line is the calculation described in the text. The scan was measured along $\mathbf{Q}=(h, h,-1.8)$ within the $(h h l)$ zone. The inset shows the scan direction within the $(h, k,-1.8)$ zone.

results in little interference. At $\mathbf{Q}_{\mathrm{AF}}$ the scattering from an individual domain does not get cancelled by the weak contributions from neighboring domains. In contrast, the situation for $\mathrm{La}_{1.85} \mathrm{Sr}_{0.15} \mathrm{CuO}_{4}$ is $\xi>n a$. That is the necessary for condition for obtaining well resolved IC peaks. Although the correlations between antiphase domains are perhaps less well defined in 123 , the smaller value of $\xi$ suggests greater stripe fluctuations, a condition that appears to correlate with an increased $T_{c}[2]$.

\section{DISCUSSION}

It has been shown that the $\mathbf{Q}$ dependence of scattered intensity from low-energy spin fluctuations in $\mathrm{La}_{1.85} \mathrm{Sr}_{0.15} \mathrm{CuO}_{4}$ and $\mathrm{YBa}_{2} \mathrm{Cu}_{3} \mathrm{O}_{6.6}$ can be consistently interpreted on the basis of dynamic antiphase antiferromagnetic domains. The correlation lengths $\xi$ used to model the scattering 
measurements were calculated from experimental values of $\Gamma$ and $\hbar c_{0}$. It was pointed out that the IC peaks become unresolvable when $\xi<n a$, where $n a$ is the charge-stripe spacing.

There are, of course, other neutron scattering results that are consistent with the stripe picture. A comparison of $\tilde{\chi}^{\prime \prime}(\omega)$ in superconducting $\mathrm{YBa}_{2} \mathrm{Cu}_{3} \mathrm{O}_{6.6}$ and antiferromagnetic $\mathrm{YBa}_{2} \mathrm{Cu}_{3} \mathrm{O}_{6.15}$ shows that, despite a difference in $\omega$ dependence, the overall weight within the range 5 to $50 \mathrm{meV}$ is comparable [14]. Similar results were found in a comparison of $\mathrm{La}_{1.85} \mathrm{Sr}_{0.15} \mathrm{CuO}_{4}$ and $\mathrm{La}_{2} \mathrm{CuO}_{4}$ 10, although the spectral weight for the superconductor is somewhat reduced when the comparison is extended up to $300 \mathrm{meV}$. With a strong segregation of holes of density $n_{h}$, the density of $\mathrm{Cu}$ moments contributing to the lowenergy $(<300 \mathrm{meV})$ spin fluctuations is roughly $1-2 n_{h}$, so one may expect to see relatively little change in overall spectral weight compared to the undoped antiferromagnet.

Measurements on $\mathrm{Zn}$-doped $\mathrm{La}_{1.86} \mathrm{Sr}_{0.14} \mathrm{CuO}_{4}$ have shown that the positions and $Q$-widths of the IC peaks are essentially unaffected by the presence of the Zn 17]. Similarly, doping Zn into $\mathrm{YBa}_{2} \mathrm{Cu}_{3} \mathrm{O}_{6.6}$ does not change the $\mathbf{Q}$ dependence of the scattering, but it does shift spectral weight down to low-energy, so that $\tilde{\chi}^{\prime \prime}(\omega)$ looks more like that of an antiferromagnet [18]. This suggests that doping $\mathrm{Zn}$ into the $\mathrm{CuO}_{2}$ planes destroys superconductivity by pinning the stripes.

Recently there has been considerable excitement over observations of resonant enhancements of the magnetic scattering below $T_{c}$ [19]20]. If the enhanced scattering is assumed to come from intinerant electrons, then it is certainly quite remarkable. On the other hand, if the magnetic scattering all comes from antiferromagnetic domains between charge stripes, the change in the scattering might in some way be associated with a reduction in damping of the spin fluctuations due to the onset of superconductivity. In any case, it will be interesting to see how models of these effects evolve.

\section{REFERENCES}

1. J. M. Tranquada et al., Nature 375, 561 (1995); J. M. Tranquada et al., Phys. Rev. B 54, 7489 (1996).

2. J. M. Tranquada et al., Phys. Rev. Lett. 73, 338 (1997).

3. C. H. Chen, S.-W. Cheong, and A. S. Cooper, Phys. Rev. Lett. 71, 2461 (1993); J. M. Tranquada, D. J. Buttrey, V. Sachan, and J. E. Lorenzo, ibid. 73, 1003 (1994).

4. S.-W. Cheong et al., Phys. Rev. Lett. 67, 1791 (1991).

5. K. Yamada et al. (preprint).

6. M. K. Crawford et al., Phys. Rev. B 44, 7749 (1991); J. D. Axe et al., Phys. Rev. Lett. 62, 2751 (1989).

7. A. Auerbach and D. P. Arovas, Phys. Rev. Lett. 61, 617 (1988); S. Tyč, B. I. Halperin, and S. Chakravarty, ibid. 62, 835 (1989); G. J. Mata and G. B. Arnold, Phys. Rev. B 39, 9768 (1989).

8. A. H. Castro Neto and D. Hone, Phys. Rev. Lett. 76, 2165 (1996).

9. J. M. Tranquada, P. Wochner, and D. J. Buttrey, cond-mat/9612007.

10. S. M. Hayden et al., Phys. Rev. Lett. 76, 1344 (1996).

11. G. Aeppli et al., Phys. Rev. Lett. 62, 2052 (1989).

12. B. J. Sternlieb and G. Shirane (unpublished); K. Yamada et al., Phys. Rev. Lett. 75, 1626 (1995).

13. J. M. Tranquada et al., Phys. Rev. B 46, 5561 (1992).

14. S. Shamoto et al., Phys. Rev. B 48, 13817 (1993).

15. S. M. Hayden et al., Phys. Rev. B 54, R6905 (1996).

16. M. R. Presland et al., Physica C 176, 95 (1991); S. D. Obertelli, J. R. Cooper, and J. L. Tallon, Phys. Rev. B 46, 14928 (1992).

17. M. Matsuda et al., J. Phys. Soc. Jpn. 62, 443 (1993).

18. K. Kakurai et al., Phys. Rev. B 48, 3485 (1993).

19. T. E. Mason et al., Phys. Rev. Lett. 77, 1604 (1996).

20. H. F. Fong, B. Keimer, D. L. Milius, and I. A. Aksay, Phys. Rev. Lett. 78, 713 (1997); P. Dai 
et al., ibid. 77, 5425 (1996). 